\title{
Step-By-Step Instructions For The Innovative Three- Port-Technique of Laparoscopic Sleeve Gastrectomy: How I Do It \
}

\section{zhiyong dong}

Jinan University First Affiliated Hospital

\section{Christine Stier}

Sana Hospitals Germany

\section{Wenhui Chen}

Jinan University First Affiliated Hospital

\section{Tsz Hong Chong}

Jinan University First Affiliated Hospital

Jingge Yang

Jinan University First Affiliated Hospital

Chetan Parmar

Whittington Health NHS Trust

Cunchuan Wang ( $\nabla$ twcc2015@163.com )

Jinan University First Affiliated Hospital

\section{Technical advance}

Keywords: Metabolic and bariatric surgery, laparoscopic sleeve gastrectomy, LSG, procedure

Posted Date: January 18th, 2021

DOI: https://doi.org/10.21203/rs.3.rs-146710/v1

License: (c) (i) This work is licensed under a Creative Commons Attribution 4.0 International License. Read Full License 


\section{Abstract}

Background Laparoscopic sleeve gastrectomy (LSG) is considered a standard bariatric procedure, which is easy to perform. However, there are pitfalls to be aware of, because complications from the operation, on the other hand, pose great challenges.

Methods We describe our innovative three-port-technique of LSG in a step-by-step instruction. To investigate the safety and efficacy, a retrospective evaluation and analysis was performed on consecutive 116 patients. For this purpose, the operation time, complication rates and weight loss were recorded.

Results Mean BMI was $35.47 \pm 4.53 \mathrm{~kg}$ Mean procedure duration was $90.13 \pm 5.68$ mins. Mean weight loss achieved was $11.57 \pm 5.09 \mathrm{~kg}$ at first month; $19.75 \pm 6.57 \mathrm{~kg}$ at third month; $26.85 \pm 7.60 \mathrm{~kg}$ at sixth month, and $28.86 \pm 11.23 \mathrm{~kg}$ at one year. According to the Clavien-Dindo classification, no seriously complications were observed after 1, 3, 612 months follow-up.

Conclusion LSG in this innovative three-port technique can be performed safely with a convincing outcome.

\section{Background}

Technically, "laparoscopic sleeve gastrectomy" describes the surgical reduction of the natural stomach volume by approximately $80-90 \%$ while leaving a tubular stomach. Initially, the large curvature of the stomach is mobilized. The dissection begins mainly $3 \mathrm{~cm}$ above the pylorus. The Aa. gastricae breves are then completely dissected and the entire fundus of the stomach up to the His angle is mobilized. Finally, the largest part of the stomach is resected along a "safety bougie", nasogastric tube of 36Fr charière, which is placed in the stomach to prevent the resulting gastric sleeve from becoming too tight, especially at the height of the angulus fold [1-3]. It appears to be a technical procedure that provides relatively simplicity and thus a flat learning curve, a short operation time and, at the same time, it avoids the implantation of a foreign body. This has led to the fact that within a short period of time the LSG has become the most frequently used bariatric procedure worldwide. [4-6]. Nevertheless, this procedure has its immanent pitfalls and thus demands personal surgical skills and an accurate and exact technical realization. We have further developed this technique and minimized surgical access by reducing the number of trocars required and have already applied it frequently. This technique will be presented in detail in a step-by-step guide and supplemented by its clinical results.

\section{Methods}

\section{Step-by-step instructions for our three-port-technique of laparoscopic sleeve gastrectomy}

\section{Patient positioning and location of the trocar ports}


Patients are positioned supine with spread legs, in shape comparable to the Chinese letter " $\square$ ". The surgeon stands between the legs of the patient. The camera guide is positioned on its right and. A further surgical assistant the left side of the patient. The assisting nurse stands at the end of the left lower limb. The two monitors are located above the patient's head and left side. Anti-embolism stockings, in order to prevent deep venous thrombosis (DVT) are always in use (Fig. 1-1).

Trocar ports: Camera trocar port is positioned at the umbilical region. Kapnoperitoneum pressure is adjusted to $12-15 \mathrm{~mm} \mathrm{Hg}$. A $5 \mathrm{~mm}$ trocar is set in the left middle clavicular line and serves as main operation port. The $12 \mathrm{~mm}$ trocar, serving as port for the stapler is set in the right middle clavicular (Fig. 2). After adjusting the position of all the trocars, the table can be angled to 30-50 degrees high or to 10-15 degrees low reverse Trendelenburg, according to the surgeon's comfort.

\section{Identifying the pylorus and dissecting the greater omentum}

After entering the abdominal cavity, a diagnostic laparoscopy is performed. Tour nurse helps to put 36-40 Fr stomach oro-gastric tube (bougie) through the mouth and sucks the stomach empty (Fig. 3-A). Dissection at the greater curvature is started around $3 \mathrm{~cm}$ from the pylorus (Fig. 3-B). Use an ultrasound knife to make an opening in the avascular area and in the middle of the gastrocolic ligament/greater omentum. After entering the omental sac, the gastrocolic ligament along the gastric wall inside the omental vascular arch of the great curvature of the stomach is further dissected (Fig. 3-C). Attention is acquired during dissecting of the omental blood vessels to prevent a damage of the right gastroepiploic artery [7].

\section{Dissection of the gastric fundus of stomach and exposing the left crus of diaphragm}

At the spleen's hilus, the gastro-colic ligament petered out into the gastro-splenic ligament, which is formed by the two peritoneal layers, which also cover the anterior and posterior gastric wall. There ligament harbours the short gastric arteries (Aa. gastricae breves), the left gastroepiploic artery and its accompanying vein. Dissection is initiated by coagulation of the short gastric vessel (Fig. 4-A). Near the left diaphragmatical angle, attention has to be paid to a short gastric artery that usually enters the stomach through the rear of the fundus close to the small curvature. This blood vessel should be dissected to ensure complete mobilization of the gastric fundus. The space between the stomach and the hilus of spleen is usually very limited. This requires special attention in order not to get too close to the stomach wall and thus compromise its blood supply, (Fig. 4-B) while simultaneously avoiding damage or bleeding from the spleen [8]. Thermal damage of the gastric wall may increase the risk of postoperative leakage [9]. Dissection is continued by separating the gastro-phrenic ligament in upward direction (Figure $4-C)$; The gastro-esophageal junction and the left phrenic curs have to be properly exposed. The resulting complete exposure of the left diaphragmatic angle thereby confirms achievement of the necessary complete mobilization of the gastric fundus. Final attention of this step requires the unconditional protection of a phrenic blood vessel, located at the basis of the phrenic muscle [10].

\section{Dissecting the posterior gastric wall}


The posterior wall of the stomach is successively dissected by moving upward off from antrum to the left crus (Fig. 5-A, B). For a proper exposure of the mobilized stomach, membranous structures between posterior gastric wall and pancreas have to be disengaged and dissected (Fig. 5-C). During this step, attention has to be paid, in order to protect the left gastric artery and its lymph nodes. Therefore, the surgeon's left-hand gastric grasping forceps pulls the gastric wall forward while assisting the exposure. In principle, the dissection level must not be too deep in order to avoid the complication of a total gastrectomy becoming necessary with impaired blood supply from the left gastric artery (Fig. 5-D), [11].

\section{Resection of the excess volume along the greater curve while forming a sleeved stomach}

The inserted oro-gastric tube is advanced into antrum as a guide for calibrating bougie. The distance of 2-6 $\mathrm{cm}$ to the pylorus of the initial stapler placement should ensure reduction of the antral volume while preserving pylorus function (Fig. 6-A). During dissection, further attention has to be paid to the resulting width of the sleeve at the incisura angularis. If here the diameter becomes too tight, this results in the most common reason for postoperative stenosis. (Fig. 6-B). Thus, it is necessary to avoid distortion with a resulting (Fig. 6-C) discrepancy between anterior and posterior wall of the stomach (Fig. 6-D). After passing these two neural points - antrum and angulus fold - the sleeve is formed further upwards under constant calibration. The position of last stapler is suggested to be placed with a distance of $0.5-1 \mathrm{~cm}$ away to the angle of His and apart of the gastroesophageal junction's fat pad, again to ensure sufficient (Fig. 6-E, F) blood supply of the upper sleeve area, in order to avoid an increased risk of leakage [12].

Selection of staple cartridge: Staple cartridges with a height not less than $2 \mathrm{~mm}$ are appropriate for the dissection from antrum level up to the angulus fold (e.g. green cartrige, Johnson \& Johnson) A height of minimal $1,5 \mathrm{~mm}$ should be selected for the dissection from the angulus fold upwards to the angle of His (e.g. blue cartrige, Johnson \& Johnson) [13]. The tissue should be kept pressed between the cartrige branches for at least 15 seconds before the stapler may be fired. With a manually stapler in use, at least 5 seconds are sufficient. In case, the left hepatic lobe is voluminous thus obstructing the surgeon's view, it may be lifted and thus retracted with the surgeon's left-hand gastric grasping forceps, therewith enabling the inspection of the staple line.

\section{Over-Sewing of Staple Line and fixation of the greater omentum to the staple line}

An additional running suture along the staple line with an absorbable threat prevents bleeding and possible even the risk of leakage (Fig. 7-A) thereby re-attaching the previously disconnected omentum (Fig. 7-B). During the over sewing, the oro-gastric tube should be kept in place, in order to prevent an inadvertent constriction of the sleeve. The use of a non-absorbable suture is not recommended, because of the risk of granuloma formation or initiation of a fistula [9]. During this step, an obstructed view by a big left hepatic lobe, the surgeon's right-hand gastric grasping forceps may help to retract the liver.

\section{Removing the resected specimen and suturing the trocar port}


The salvage of the specimen is the last step of the procedure. Attention must be paid to avoid damage and contamination of the incision. This step can be intraabdominal monitored with the camera. Intraluminal conditions, such as bleeding, can be monitored with Intraoperative gastroscopy and gas insufflation can be used to detect leakage. The leak test is performed as "bicycle tire test" by flooding the sleeve with water while insufflating the gastric lumen with gas during endoscopy. Rising bubbles would indicate a leak.

After this step, the $12 \mathrm{~mm}$ Trocar is removed under direct vision to exclude local bleeding. Insertion of an intraabdominal drainage is optional. Under laparoscopic monitoring, the $12 \mathrm{~mm}$ trocar hole is closed in order to prevent trocar site hernia and then the remaining trocars are also removed under sight and the incisions are closed with sutures [14].

\section{Results}

In addition to the description of the surgical laparoscopic three-port-technique, the retrospectively analyzed results of the116 patients will be presented. All surgeries were performed between June 2009 and July 2019. The procedure was completed in 119 patients with this reduced-port-technique.

Complication rate:

The complications that occurred in the 116 cases described were analyzed according to the ClavienDindo Classification. Only minor complications, classified as Class I (6 / 5.1\%) occurred. No major complications (Class II, III, IV, V) were observed. There was also no lethal outcome within the first 30 postoperative days in any of the patients (mortality rate: $0 \%$ ). At one year none of the patients reported about a new onset of complaints eg. reflux, regurgitation, or vomiting.

Conversion rate to a more-than-three-port technique:

In two cases an additional port was necessary to complete the operation. This number corresponded to $1.7 \%$. There were various reasons for this, which are listed below: large liver volume, abdominal space, and abdominal fat.

Bariatric outcome:

All patients were followed up after 1,3,6, and 12 months. Accordingly, 98.3\%, 96.6\% and $95.6 \%$ of the patients could be evaluated at these points in time. The results of total body weight loss in $\mathrm{kg}$ (TBWL) and of excess weight loss (EWL) are presented in the Table 1. 
Table 1

Weight changes $1,3,6$ and 12 months years after surgery

\begin{tabular}{|llllll|}
\hline & Base line & 1 month & 3 months & 6 months & 12 months \\
\hline Number & 116 & 114 & 112 & 112 & 111 \\
\hline BMl $\left(\mathrm{kg}\left(\mathrm{m}^{2}\right)\right.$ & $35.47 \pm 4.53$ & $31.41 \pm 3.95^{\star}$ & $28.46 \pm 382^{*}$ & $25.89 \pm 3.80^{*}$ & $25.13 \pm 4.20^{*}$ \\
\hline Weight $(\mathrm{kg})$ & $98.26 \pm 18.40$ & $86.93 \pm 16.25^{\star}$ & $78.64 \pm 14.83^{*}$ & $71.56 \pm 14.16^{\star}$ & $69.61 \pm 15.47^{*}$ \\
\hline TWL $(\mathrm{kg})$ & & $11.57 \pm 5.09$ & $19.75 \pm 6.57$ & $26.85 \pm 7.60$ & $28.86 \pm 11.23$ \\
\hline EWL $(\%)$ & & $44.47 \pm 21.50$ & $77.59 \pm 35.34$ & $103.81 \pm 43.13$ & $109.45 \pm 70.86$ \\
\hline TWL: total weight los EWL: excess weight loss & & & \\
\hline
\end{tabular}

\section{Discussion}

LSG in this innovative three-port technique was proofed to be safe with a convincing outcome in a Chinese cohort with 1300 patients, performed in an academic hospital setting.

In a worldwide comparison, Chinese patients show to have a lower BMI when undergoing bariatric surgery, here $35.47 \pm 4.53$. This can be an advantage with this surgical technique. However, it has been shown with our cohort that a higher BMI is no obstacle to this new technology using a smaller number of ports. The purely bariatric result in terms of weight loss and excess weight loss stands up to comparison with the results of LSG performed in conventional technology with 4-5 ports. The difference between the three-port technique and the conventional technique is that no additional liver retractor is required. If necessary, the left lobe of the liver is lifted by the surgeon grasper, either acting with the left or righthanded positioned instrument, as needed. This technique is easy to learn and in our experience the learning curve is already completed with 20 interventions. If the left hepatic lobe is so voluminous that it would be necessary to lift it permanently for an unobstructed view, another port can be added after inspection of the situs. Thus, the operation planning is basically carried out for the three-port technology and only if necessary, a further port is added. Our cohort has shown that this was only necessary in very few cases.

This study is limited by the fact that it was a retrospective evaluation and that the results represent a single-institution experience.

\section{Conclusion}

To sum up, according to the study, LSG in this innovative three-port technique can be performed safely with a convincing outcome.

\section{Abbreviations}


LSG: laparoscopic sleeve gastrectomy; DVT: deep venous thrombosis; TBWL: total body weight loss; EWL: excess weight loss; BMI: body mass index

\section{Declarations}

\section{Ethics approval and consent to participate}

This study was reviewed and approved by the institutional Ethical Board of First Affiliated Hospital of Jinan University. And the need for informed consent was waived due to the retrospective nature of the study.

\section{Consent for publication}

Not applicable

\section{Availability of data and materials}

The datasets used and/or analysed during the current study available from the corresponding author on reasonable request.

\section{Competing interests}

The authors declared no competing interests.

\section{Funding}

This work was not funded.

\section{Authors' contributions}

CW, DZ and SC designed the study. ZD, DZ, CW and PC drafted the manuscript. ZD, SC, CW, CT and YJ extracted the data and analysis the selected papers. All authors reviewed and approved the final version of this paper.

\section{Acknowledgements}

Not applicable

\section{References}

1. Ali M, El Chaar M, Ghiassi S, et al. American Society for Metabolic and Bariatric Surgery updated position statement on sleeve gastrectomy as a bariatric procedure. Surg Obes Relat Dis, 2017, 13(10): 1652-1657.

2. Gagner M, Hutchinson C, Rosenthal R. Fifth International Consensus Conference: current status of sleeve gastrectomy. Surg Obes Relat Dis, 2016, 12(4): 750-756. 
3. Rosenthal RJ, Diaz AA, Arvidsson D, et al. International Sleeve Gastrectomy Expert Panel Consensus Statement: best practice guidelines based on experience of $>12,000$ cases. Surg Obes Relat Dis, 2012, 8(1): 8-19.

4. Khidir N, El-Matbouly MA, Sargsyan D, et al. Five-year Outcomes of Laparoscopic Sleeve Gastrectomy: A Comparison Between Adults and Adolescents. Obes Surg, 2018, 28(7): 2040-2045.

5. Dong Z, Islam SMS, Yu AM, et al. Laparoscopic metabolic surgery for the treatment of type 2 diabetes in Asia: a scoping review and evidence-based analysis. BMC Surg, 2018, 18(1): 73.

6. Karmali S, Johnson stoklossa C, Sharma A, et al. Bariatric surgery: a primer. Can Fam Physician. 2010;56(9):873-9.

7. Clapp B. Anatomic landmarks in the sleeve gastrectomy. JSLS. 2013;17(3):388-389. doi:10.4293/108680813X13654754534152

8. Jossart GH. Complications of sleeve gastrectomy: bleeding and prevention[J]. Surg Laparosc Endosc Percutan Tech, 2010, 20(3): 146-147.

9. Kourosh Sarkhosh, MD, MSc, Daniel W. Birch, MD, MSc, Arya Sharma, MD, PhD, DSc, and Shahzeer Karmali, BSc, MD Complications associated with laparoscopic sleeve gastrectomy for morbid obesity: a surgeon's guide. Can J Surg. 2013 Oct; 56(5): 347-352.

10. Splenic infarct as complication of sleeve gastrectomy. Surgery for Obesity and Related Diseases 5 (2009) 626-629.

11. Laparoscopic Sleeve Gastrectomy with an Extensive Posterior Mobilization: Technique and Preliminary Results. August 20110besity Surgery 22(2):320-9

12. Ortega CB, Guerron AD, Yoo JS. The Use of Fluorescence Angiography During Laparoscopic Sleeve Gastrectomy. JSLS. 2018;22(2):e2018.00005.

13. Moon RC, Shah N, Teixeira AF, Jawad MA. Management of staple line leaks following sleeve gastrectomy. Surg Obes Relat Dis. 2015;11(1):54-59

14. Nguyen N T , Blackstone R P, Morton J M , et al. The ASMBS Textbook of Bariatric Surgery II 2015.

\section{Figures}




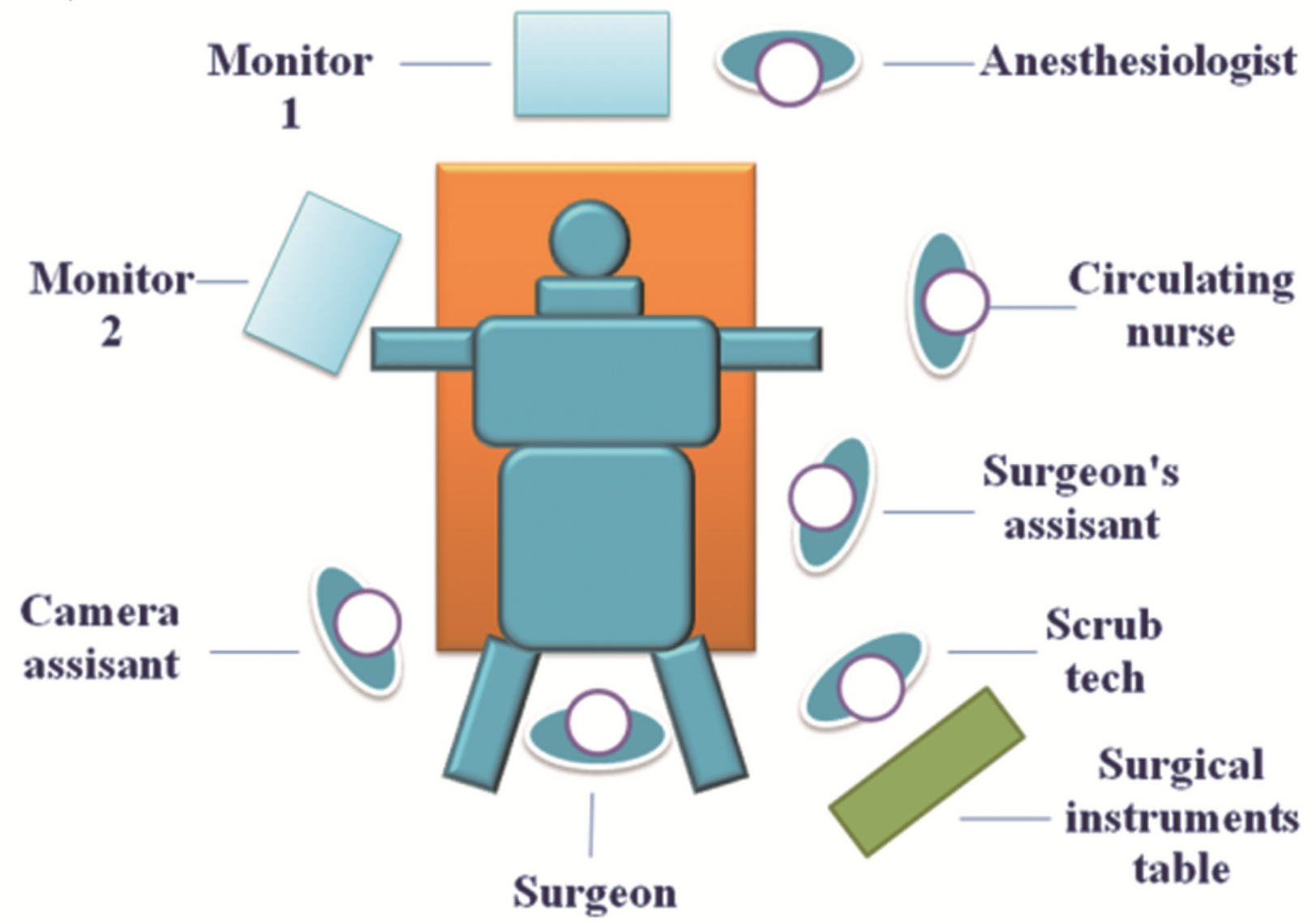

Figure 1

Position of patient and surgeon 


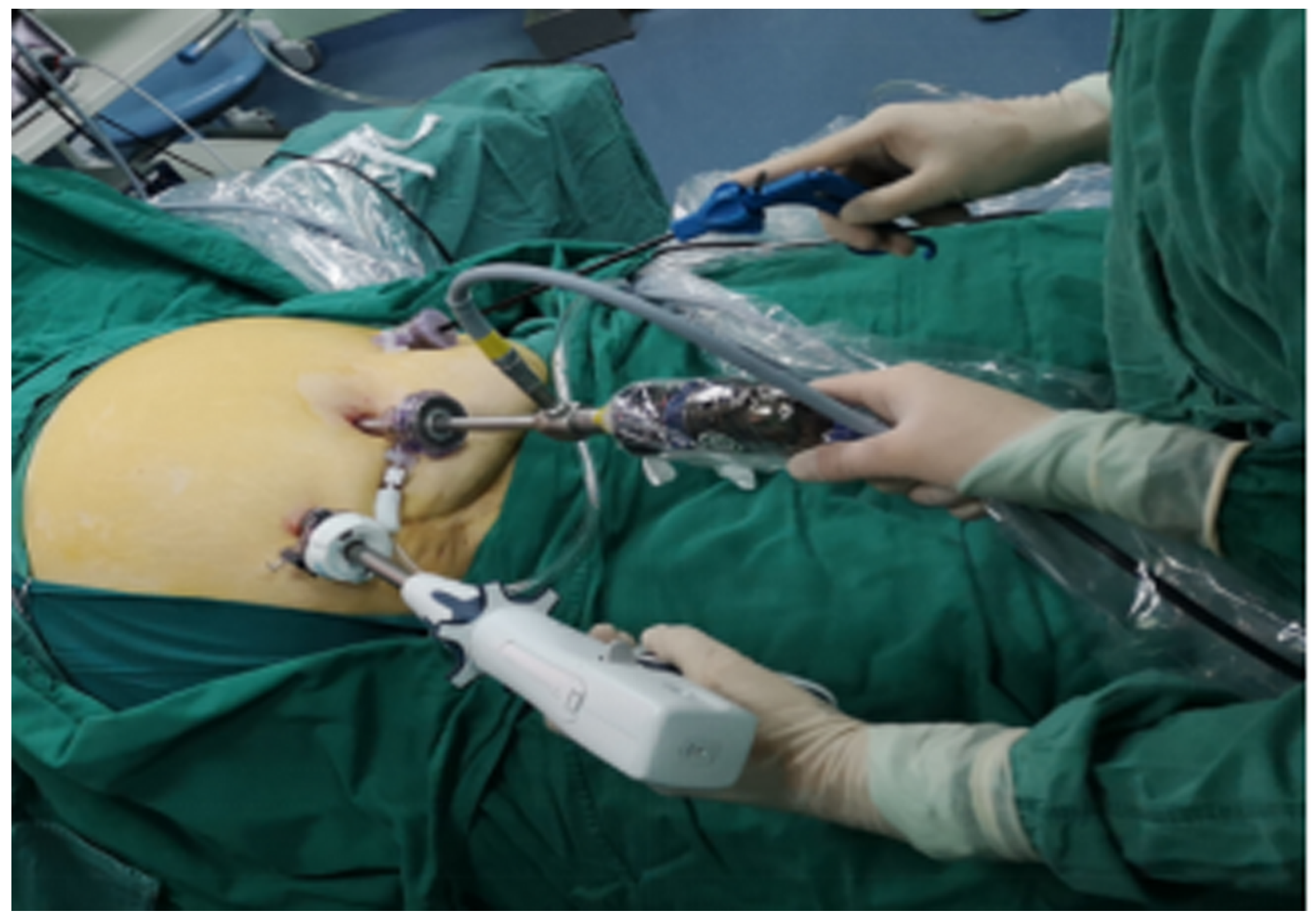

Figure 2

Trocar position: a $10 \mathrm{~mm}$ trocar is placed $1.0 \mathrm{~cm}$ below the umbilicus as the camera/observation port, a 5 $\mathrm{mm}$ trocar is placed at the mid clavicular line as the main operation port, and a $12 \mathrm{~mm}$ trocar is placed at the right mid clavicular line as the auxiliary operation hole (three port technique). 


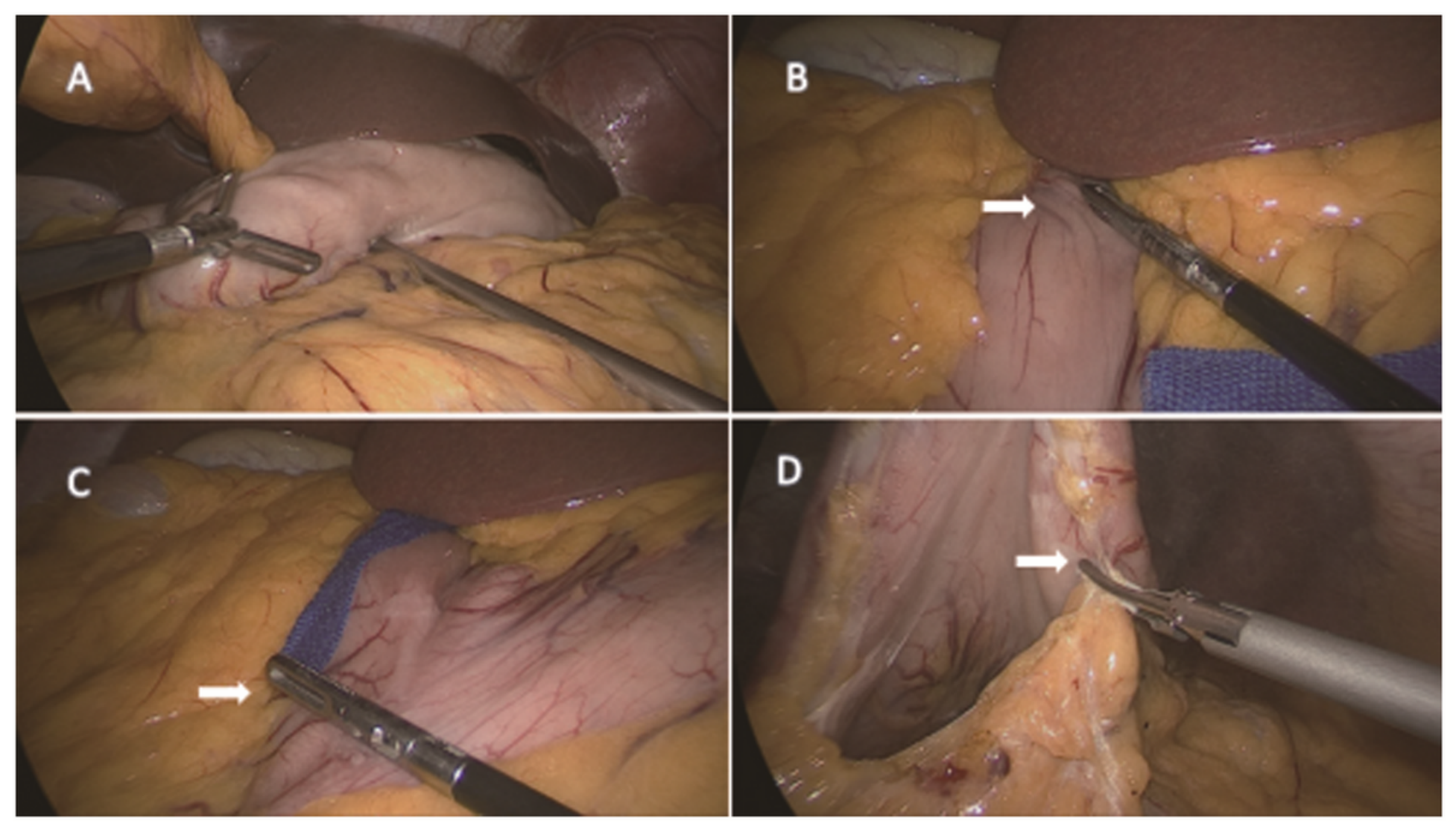

\section{Figure 3}

Identifying the pylorus and dissecting the greater omentum: A. put a 36-40fr bougie, make the stomach empty; B. Identifying the pylorus (arrow: anterior pylorus vein); C measure the boundary mark of the greater omentum; $D$ dissecting the gastrocolic ligament inside the gastro-omental vascular arch (arrow: greater curvature of the stomach). 


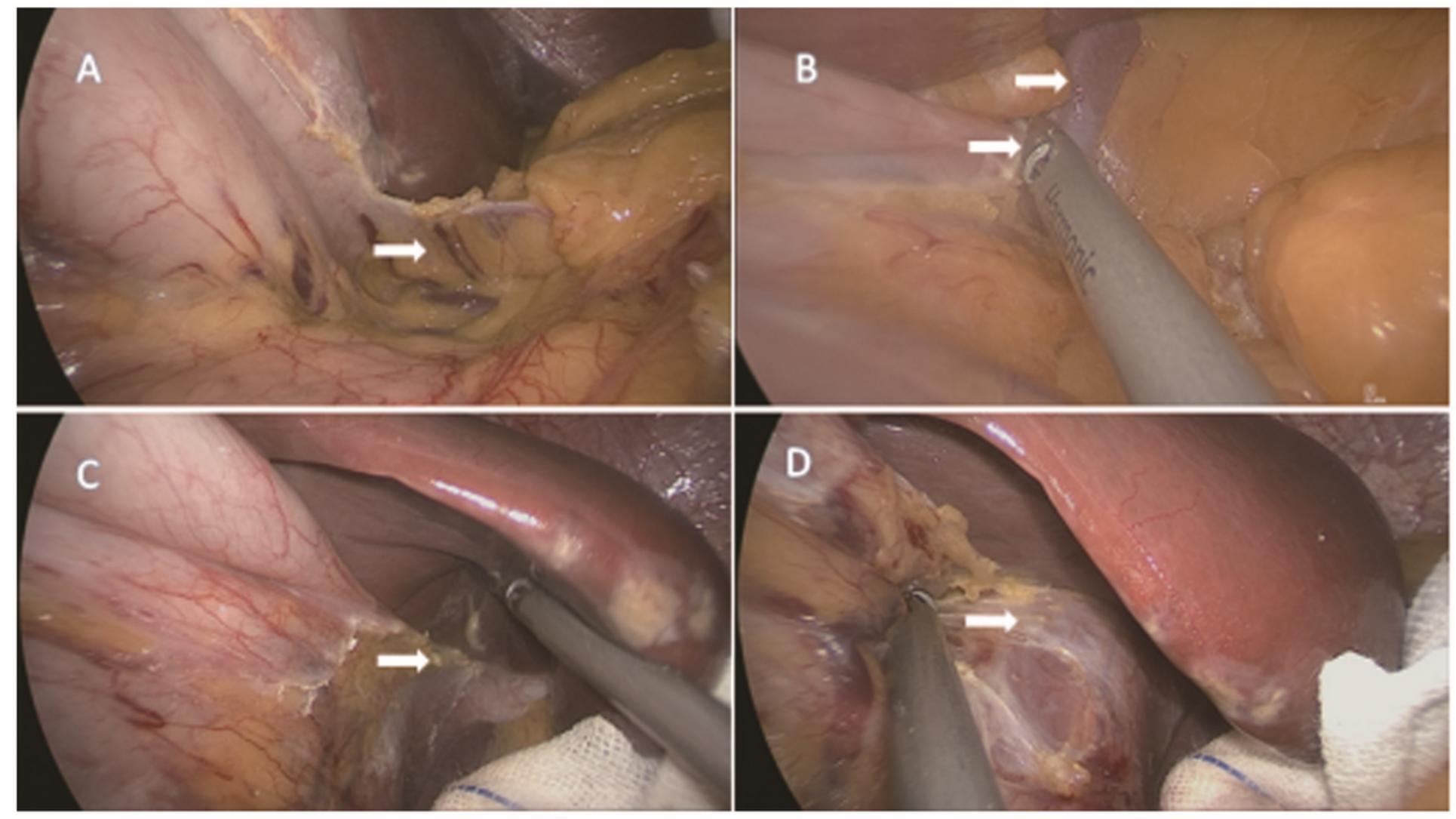

\section{Figure 4}

Dissociating the fundus of stomach and exposing the left crus of diaphragm: coagulate the short gastric blood vessels (arrow: short gastric blood vessels); B separate spleen and stomach ligaments (up arrow: spleen, down arrow: spleen and stomach ligaments); C separate stomach and diaphragm ligaments (arrow: stomach and diaphragm ligaments); D: expose left phrenic foot (arrow head: left phrenic foot) 


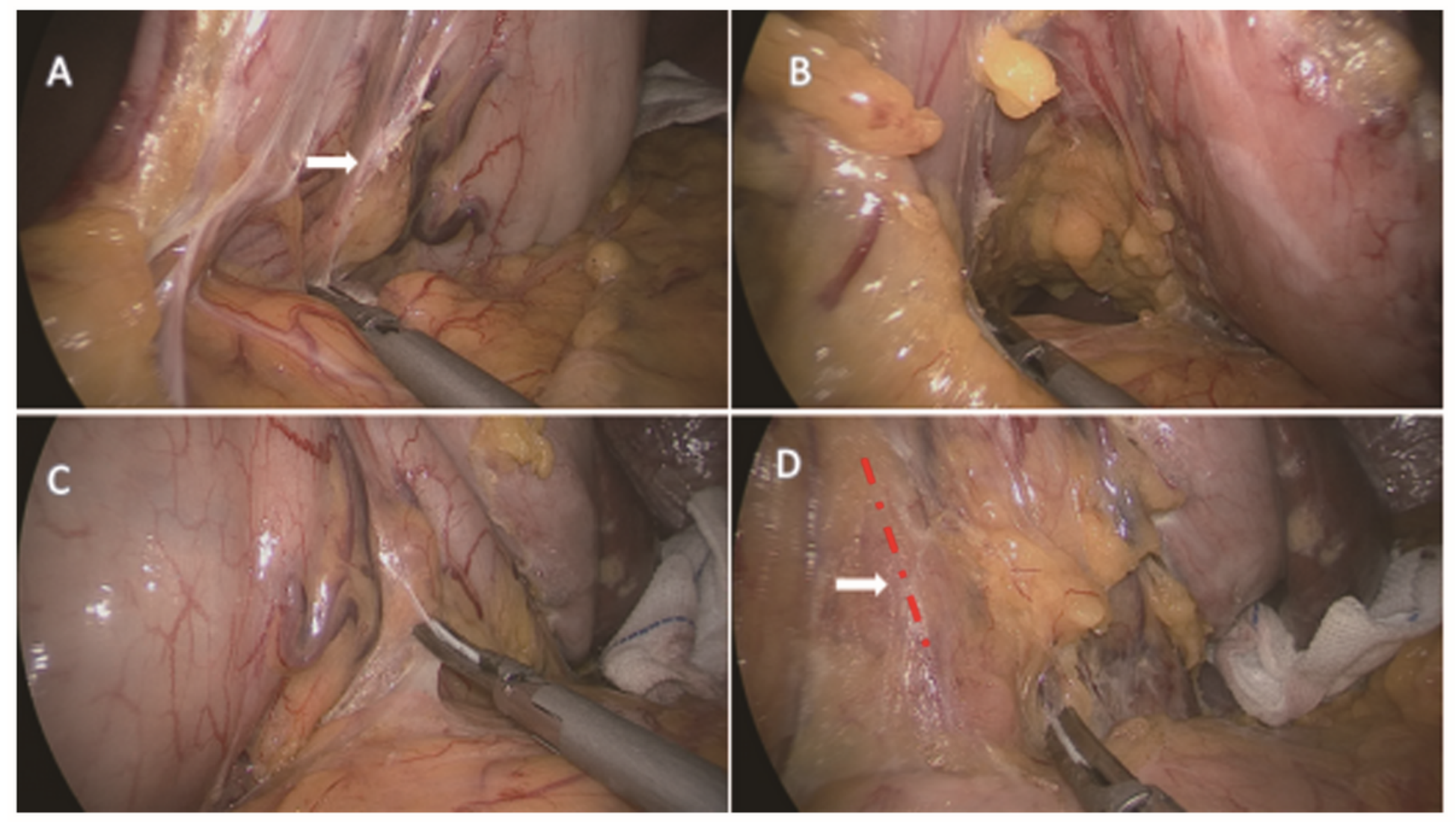

\section{Figure 5}

Dissociating posterior gastric wall: dissociating posterior gastric wall on the side of pylorus (arrow: posterior gastric wall); B completed dissociating posterior gastric wall on the side of pylorus; $\mathrm{C}$ open the gap between the posterior gastric wall and the surface of pancreas; D completed dissociation of posterior gastric wall (arrow: left gastric vascular shadow) 

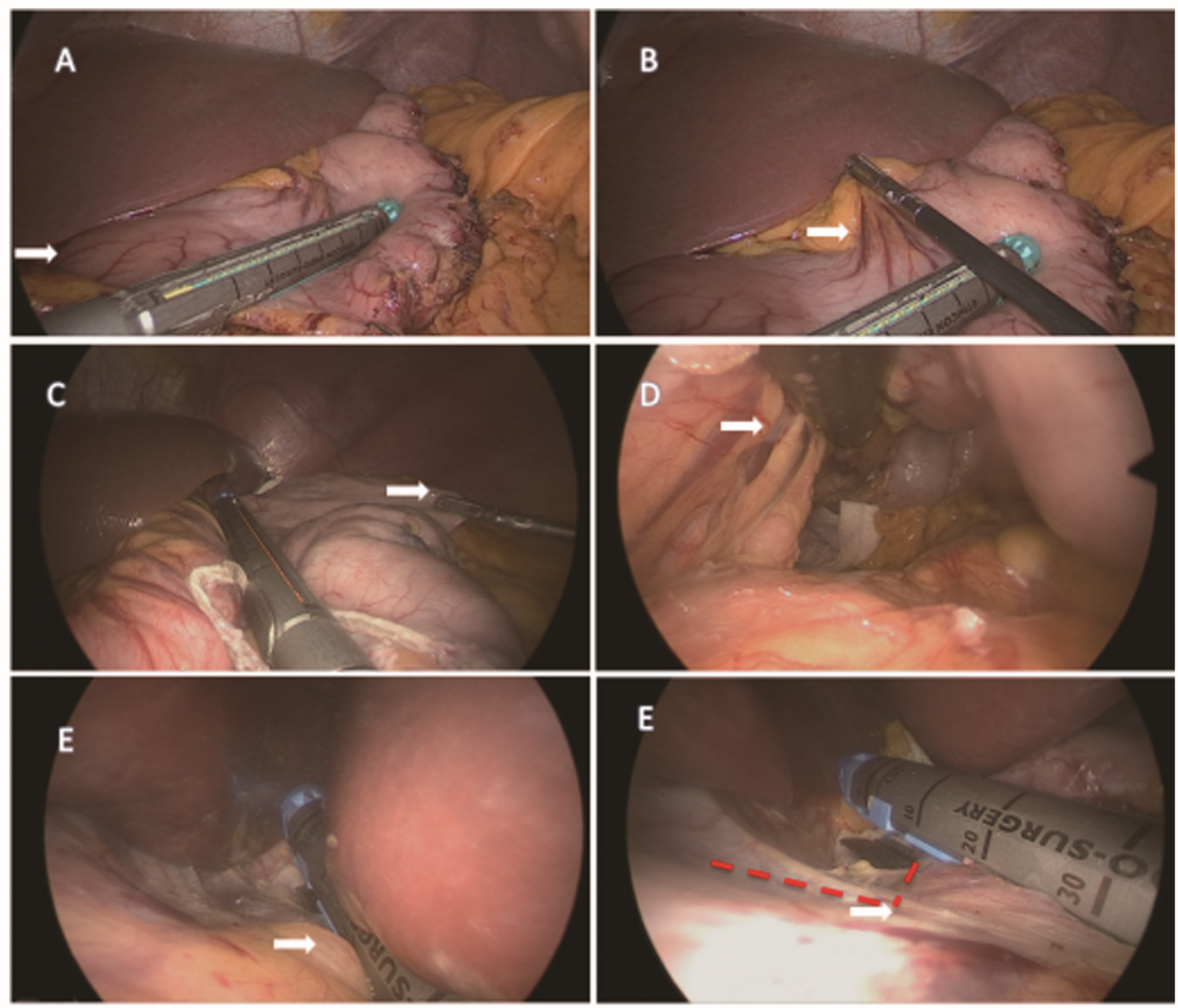

\section{Figure 6}

cutting of the great curvature of the stomach and making sleeve: $A$ is $3-4 \mathrm{~cm}$ away from the pylorus as the starting point (arrow: pylorus); $B$ is properly away from the incision of the gastric angle (arrow: the incision of the gastric angle); $C$ is laterally pulling the great curvature of the stomach and flattening the gastric wall (arrow: the operation forceps of laterally pulling); $D$ is to observe whether the posterior wall of the stomach is flattened (arrow: the posterior wall of the stomach); $E$ is to cut outside the fat pad of the gastroesophageal junction (Arrow: fat pad of gastroesophageal junction); $F$ is $1 \mathrm{~cm}$ away from his angle to complete cutting (arrow: his angle) 


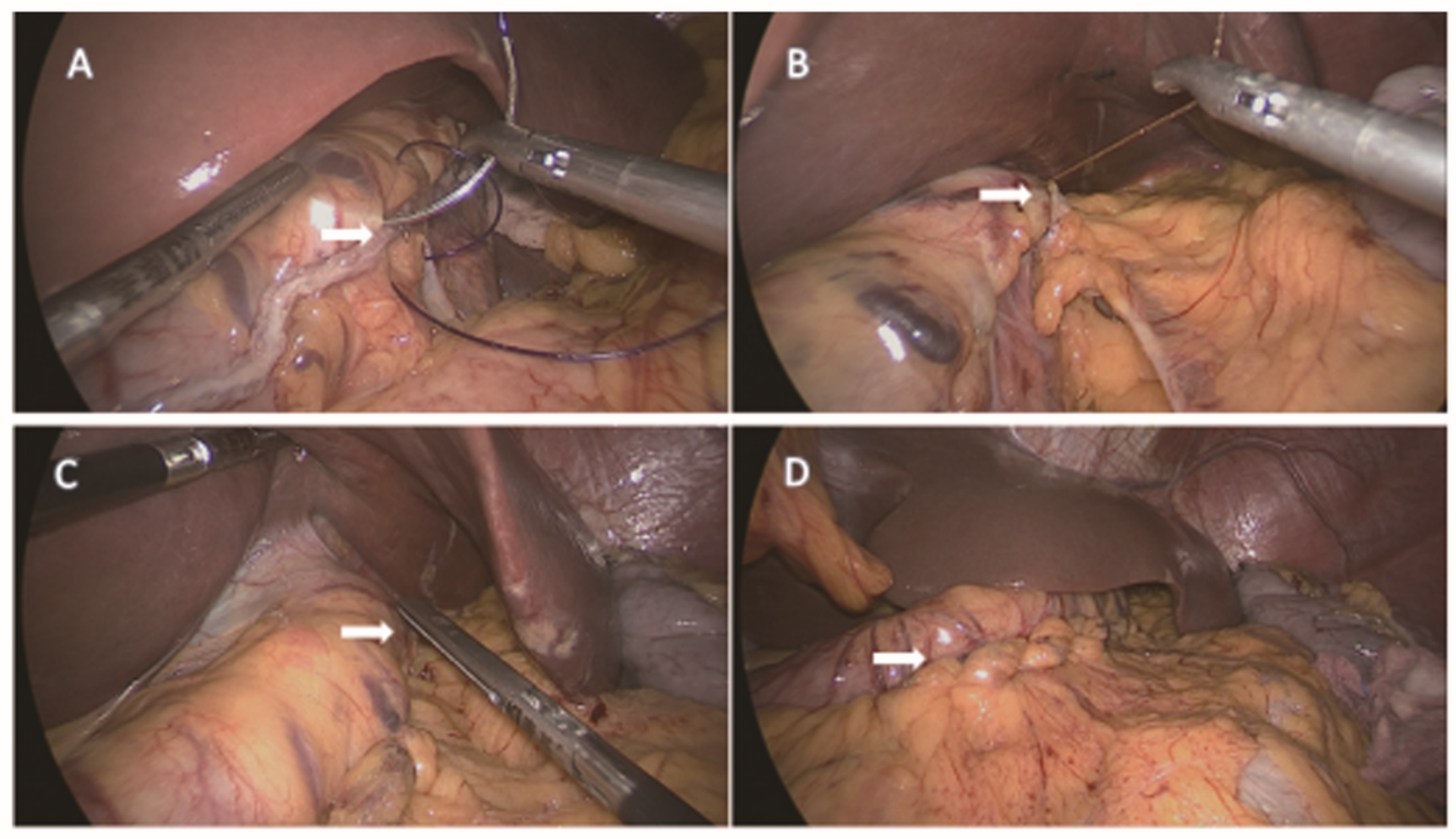

\section{Figure 7}

Over-Sewing of Staple Line and suturing the greater omentum and staple line : A continuous reinforcement of the suture sarcoplasmic layer (arrow: the incision margin of the stomach); B suture reduction of the greater omentum; $C$ the upper margin of the greater omentum after suture; $D$ the lower margin of the greater omentum after suture (arrow: reset, the incision margin of the stomach - the greater omentum) 